\title{
Movement and evolution of macromolecules in a grooved micro-channel
}

\author{
L.W. Zhou ${ }^{1}$, M.B. Liü ${ }^{* 1}$ and J.Z. Chang ${ }^{2}$ \\ ${ }^{1}$ Institute of Mechanics, Chinese Academy of Sciences, Beijing 100190, China \\ ${ }^{2}$ School of Mechatronic Engineering, North University of China, Taiyuan 030051, China
}

(Received March 10, 2013, Revised April 1, 2013, Accepted May 6, 2013)

\begin{abstract}
This paper presented an investigation of macromolecular suspension in a grooved channel by using the dissipative particle dynamics (DPD) with finitely extensible non-linear elastic (FENE) bead spring chains model. Before studying the movement and evolution of macromolecules, the DPD method was first validated by modeling the simple fluid flow in the grooved channel. For both simple fluid flow and macromolecular suspension, the flow fields were analyzed in detail. It is found that the structure of the grooved channel with sudden contraction and expansion strongly affects the velocity distribution. As the width of the channel reduces, the horizontal velocity increases simultaneously. Vortices can also be found at the top and bottom corners behind the contraction section. For macromolecular suspension, the macromolecular chains influence velocity and density distribution rather than the temperature and pressure. Macromolecules tend to drag simple fluid particles, reducing the velocity with density and velocity fluctuations. Particle trajectories and evolution of macromolecular conformation were investigated. The structure of the grooved channel with sudden contraction and expansion significantly influence the evolution of macromolecular conformation, while macromolecules display adaptivity to adjust their own conformation and angle to suit the structure so as to pass the channel smoothly.
\end{abstract}

Keywords: dissipative particle dynamics; macromolecular suspension; grooved micro-channel

\section{Introduction}

Understanding the dynamic behavior of macromolecules, such as DNA, is very important for fundamental research and practical applications in bio, chemical and medical engineering, especially in designing micro-devices. Recently, micro-devices enable processing, analyzing, and delivering biochemical materials in a wide range of biomedical and biological applications (Chun et al. 1999, Fan et al. 2003). For example, micro-needle can be used to efficiently and precisely deliver a small amount of drug or DNA into local tissue, skin regions, and even cells. In order to avoid pain and tissue traumas caused by traditional technologies of drug injection and delivery, a variety of micro-needles have been designed for hypodermic injection and transdermal drug delivery (Brazzle et al. 1999, Lin and Pisano 1999). Micro-channels are the main field to deliver and control injected materials. By designing optimal structures of micro-channels or micro-channel networks, it is possible to efficiently control the injection process, either for simple

*Corresponding author, Professor, E-mail: liumoubin@imech.ac.cn 
fluids or complex fluids with macromolecules. It is therefore very important to understand the dynamic behavior of macromolecular when passing though micro-channel with different structures.

Recent development of experimental techniques enables us to study the dynamics and rheological properties of macromolecules such as DNA in micro-channels. For example, it is possible to use fuorescence imaging techniques to visualize the micro-structural conformations of molecules (Chu 1991). Optical tweezers have been used to measure the extension properties of single DNA molecules (Perkins et al. 1994, Perkins et al. 1994). By using these techniques, some experimental works have been conducted to study the mechanics of macromolecular suspension flows. Perkins et al. (1995) measured the extension properties of tethered single DNA molecules in a uniform flow. Perkins et al. (1997), Smith and Chu (1998) studied the dynamic behavior of single DNA molecules in an elongation flow. Smith et al. (1999) observed the dynamic behavior of single DNA molecules in steady shear flows. The flow of molecular suspensions through a micro-channel is more complicated as it is a combination of non-uniform elongation and shear flows. Shrewsbury et al. (2001) used epi-fluorescence microscopy to characterize the flow's impact on the conformation of the molecules in microfluidic devices in which the path consists of a large, inlet reservoir connected to a long, rectangular channel followed by a large downstream reservoir. In the device, DNA molecules were observed to undergo elongation, non-uniform shear and compression. Near the channel wall, high shear rates results in dramatic stretching of the molecules, and may also result in chain scission of the macromolecules.

On the other hand, with the development of computational methods and computer hardware, numerical simulations of the movement and evolution of complex fluids in micro-devices have been more and more popular. Numerical simulation can provide more details on the flow field and conformations of macromolecules by tracking each molecular chain segment. In this area, the Brownian dynamics simulation (BDS) (Groot and Warren 1997) is one most common approach. Various molecular models have been used to model the DNA molecules, such as the Kramer's bead-rod chain (Doyle and Shaqfeh 1998), the FENE chain (Fan et al. 2003) and the worm-like chain in BDS (Hur et al. 2000). However, these models are usually only valid for simple fluid flow since the flow field has to be specified a-priori in BDS, such as the freely-draining flexible polymers in steady linear flow (Doyle 1997), bead-rod chains in start-up of extensional flow (Doyle and Shaqfeh 1998) and single DNA molecule in shear flow (Doyle et al. 1998).

The dissipative particle dynamics was initially designed by Hoogerbrugge and Koelman (1992), which combines features from molecular dynamics (MD) and lattice-gas automata. It aim at simulating hydrodynamic phenomena without the lattice artifacts in lattice gas automata and it can deal with much larger hydrodynamic time and space scales than MD. As a mesoscale simulation method, it is promising in simulating the movement and evolution of macro-molecules passing through micro-channels, since the flow domain is of the same order in size as macro-molecules while the continuum mechanics may not be valid. Although there are many other mesoscale simulation methods, such as BDS, Lattice Gas Automata (LGA) (Frisch et al. 1986), and Lattice Boltzmann (LB) (Chen and Doolen 1998), it is difficult for those methods to deal with complex fluids and complex flows.

Grooved channels with regular contraction and expansion sections are frequently used in micro-devices and the movement and evolution of macro-molecules are not yet not fully understood. Except for the experimental work by Shrewsbury, there are only a few works available on this subject so far. Kasiteropoulou et al. (2011) used DPD method to model simple fluid flow in periodically grooved micro-channels and to seek further insight on the effects of wall roughness on 
fluid flow. Fan et al. (2003) simulated the suspension flows of macro-molecules with FENE chain and worm-like chain through simple a micro slit, and found varieties of conformations of macro-molecular in micro-channels, including periodic contraction-diffusion micro-channels. Liu et al. (2008) used DPD to simulate electro-osmotic flow and DNA molecular in simple and complex two-dimensional and 3D geometries frequently used in nano-fluidic devices, and the results are in very good agreement with theoretical results. Pan et al. (2010) used DPD with worm-like chain to model the process of DNA separation in a micro-device, and found that longer DNA strands move faster than shorter ones.

In the present work, the DPD is used to model simple fluid and macromolecular suspension passing through the grooved micro-channels with contraction and expansion structure. The paper is organized as follows. In Sections 2 and 3, DPD methodology and macromolecular model are described respectively. In Section 4, numerical simulations of simple fluid and macromolecular suspension passing through the grooved micro-channels are analyzed. The paper concludes in Section 5 with some remarks.

\section{Methodology of the dissipative particle dynamics}

In a DPD system, a particle represents a cluster of molecules or atoms. The forces between particles are assumed to be pair-wise additive. The motion of DPD particles is governed by Newton's equations of motion. For a simple DPD particle $i$, we have the following governing equations

$$
\begin{gathered}
\frac{d \mathbf{r}_{i}}{d t}=\mathbf{v}_{i} \\
\frac{d \mathbf{v}_{i}}{d t}=\sum_{j \neq i}^{N} \mathbf{f}_{i j}+\mathbf{f}_{i}^{e x t}
\end{gathered}
$$

where $\mathbf{r}_{i}$ and $\mathbf{v}_{i}$ denote the position and velocity of particle $i$. The masses of DPD particles are usually taken to be the same as unity; and $\mathbf{f}_{i j}$ denotes the total force between particles $i$ and $j$. $\mathbf{f}_{i}^{e x t}$ is the external force, such as the gravity. The inter-particle force $\mathbf{f}_{i j}$ consists of three parts, namely: conservative force $\mathbf{F}_{i j}^{C}$, dissipative force $\mathbf{F}_{i j}^{D}$ and random force $\mathbf{F}_{i j}^{D}$

$$
\mathbf{f}_{i j}=\mathbf{F}_{i j}^{C}+\mathbf{F}_{i j}^{R}+\mathbf{F}_{i j}^{D}
$$

The conservative force describes the thermodynamic behavior of the DPD system, and can be derived form a pair potential that acts between particles $i$ and $j$ as

$$
\mathbf{F}_{i j}^{C}=\left\{\begin{array}{cc}
a_{i j}\left(1-r_{i j} / r_{c}\right) \hat{\mathbf{r}}_{i j} & r_{i j}<r_{c} \\
0 & r_{i j} \geq r_{c}
\end{array}\right.
$$


where $a_{i j}$ is the repulsion parameter between particles $i$ and $j$ represents the strength of the collision; $\mathbf{r}_{i j}=\mathbf{r}_{i}-\mathbf{r}_{j}$ represents the relative position between particle $i$ and particle $j ; r_{i j}=\left|\mathbf{r}_{i j}\right|$, $\hat{\mathbf{r}}_{i j}=\mathbf{r}_{i j} / r_{i j}$ is the unit vector directed from the mass center of particle $j$ to $i$; and $r_{c}$ is the cut-off radius. Eq. (4) indicates that the conservative force is repulsive over a limited radius $r_{c}$ only and acts in the direction of the $\hat{\mathbf{r}}_{i j}$ vector. The soft conservative force makes it possible to use a large time step in a discrete algorithm for integrating the equations of motion. The dissipative force describes for the viscous effects in the DPD system and it acts like a viscous damper by reducing the relative velocity between DPD particles. The random force represents the thermal motion of unresolved scales, such as the molecules inside each particle. The dissipative force and random force are written as

$$
\begin{gathered}
\mathbf{F}_{i j}^{D}=-\gamma \omega^{D}\left(r_{i j}\right)\left(\hat{\mathbf{r}}_{i j} \cdot \mathbf{v}_{i j}\right) \hat{\mathbf{r}}_{i j} \\
\mathbf{F}_{i j}^{R}=\sigma \omega^{R}\left(r_{i j}\right) \zeta_{i j} \hat{\mathbf{r}}_{i j}
\end{gathered}
$$

where $\gamma$ and $\sigma$ are two coefficient which represents the amplitude of the dissipative and random force, $\omega^{D}\left(r_{i j}\right)$ and $\omega^{R}\left(r_{i j}\right)$ are two weight functions which describe the variation of the friction coefficient with distance. $\mathbf{v}_{i j}\left(=\mathbf{v}_{i}-\mathbf{v}_{j}\right)$ represents the relative velocities between particle $i$ and particle $j . \zeta_{i j}$ is a random variable with Gaussian statistics

$$
\left\langle\zeta_{i j}(t)\right\rangle=0,\left\langle\zeta_{i j}(t) \zeta_{k l}\left(t^{\prime}\right)\right\rangle=\left(\delta_{i k} \delta_{j l}+\delta_{i l} \delta_{j k}\right) \delta\left(t-t^{\prime}\right)
$$

where $\delta\left(t-t^{\prime}\right)$ is a Dirac delta function, which is infinite at position $t^{\prime}$ and zero everywhere at other positions, while the integral of the Dirac delta function is unity over the entire domain. $\langle x\rangle$ represents an average value over the distribution of the random elements $x$.

As discussed by Espanol and Warren (2005), $\omega^{D}\left(r_{i j}\right)$ and $\omega^{R}\left(r_{i j}\right)$ and the corresponding coefficients $(\gamma$ and $\sigma$ ) must satisfy two requirements for correct isothermal balance

$$
\begin{aligned}
\omega^{D}\left(r_{i j}\right) & =\left[\omega^{R}\left(r_{i j}\right)\right]^{2} \\
\sigma^{2} & =2 \gamma \kappa_{b} T
\end{aligned}
$$

where, $\kappa_{b}$ is the Boltzmann constant and $T$ is the absolute temperature. There are different forms of $\omega^{D}\left(r_{i j}\right)$ and $\omega^{R}\left(r_{i j}\right)$, while a general form proposed by Fan et al. (2006) is given as follows 


$$
\omega^{D}\left(r_{i j}\right)=\left[\omega^{R}\left(r_{i j}\right)\right]^{2}=\left\{\begin{array}{cc}
\left(1-r_{i j} / r_{d}\right)^{s} & r_{i j}<r_{c} \\
0 & r_{i j} \geq r_{c}
\end{array}\right.
$$

where $r_{d}$ is the cut-off distance for the diffusive and random forces and it may not be different from $r_{c}$, which is the cut-off distance for conservative force. The choice of $r_{d}$ affects the number of pairs of interacting particles and the computation cost; the choice of affects the strength of dissipative force between particles and viscosity and Schmidt number. The common choices were $s=2$ and $r_{c}=r_{d}=1$. In this paper, we adopt $s=0.5$ and $r_{c}=r_{d}=1$.

\section{The macromolecular model}

In the DPD model, macromolecules (such as DNA molecules) are generally simulated by a series of particles (beads) linked together using springs. A variety of numerical models have been developed, such as the worm-like chain (WLC) model and FENE (finitely extendable nonlinear elastic) model. For example, Smith et al. (1999) observed a direct mechanical measurement of the elasticity of single DNA molecules by using magnetic beads; Perkins et al. (1994) directly conducted the tube-like motion of a single DNA molecule in a concentrated, $\lambda$-phage DNA solution; Perkins et al. (1995) measured the extension of a tethered DNA molecule in a uniform flow. In this paper, the FENE chain (Fan et al. 2003) is used to model macromolecules. The beads in the chain are replaced by DPD particles. In addition to the three kinds of force in Eq. (3), the spring force will also act on these beads. The spring force law of a FENE chain segment can be expressed as the following equation

$$
\mathbf{F}_{i j}^{S}=-\frac{H \mathbf{r}_{i j}}{1-\left(r_{i j} / r_{m}\right)^{2}}
$$

Where $H$ is the spring constant. $r_{m}$ is the maximum length of one FENE chain segment. From Eq. (11), we can see that the spring force increases intensely and approaches infinity when $r_{i j} / r_{m}$ approaches 1 . As a result, the distance between two neighboring beads in FENE chain should be less than $r_{m}$.

\section{Simple and complex flows in a grooved micro-channel}

In this paper, we are interested in simulating the macromolecular suspension flow through a grooved micro-channel with sudden contraction and expansion sections. When studying the transport and conformation of macromolecules, the length of the macromolecules is at the same scale as the width of micro-channels, and therefore continuum mechanics based theories may not be valid. On the other hand, the size of flow field and macromolecules, such as DNA, are too large to be handled by MD simulation, in which computational requirements exceed the capabilities of 


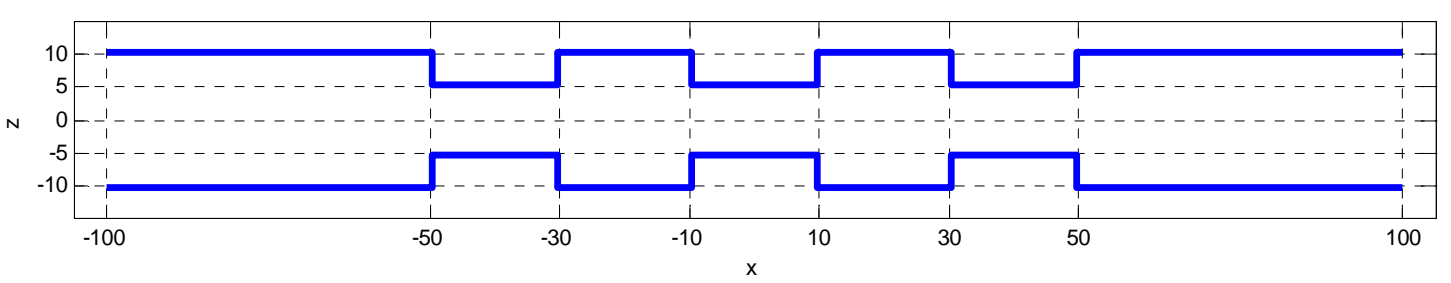

Fig. 1 Schematic view of the grooved micro-channel simulated. The computational domain is divided into 400 bins in the $\mathrm{x}$-direction, one bin in $\mathrm{y}$-direction, and 94 bins in z-direction

present-day supercomputers. As a mesoscale modeling technique, DPD can be used to predict the material properties that depend on structures and behavior between the microscopic (atomistic) and macroscopic (continuum) scales. Thus, DPD is very suitable for simulating the transport and conformation of macromolecules in grooved micro-channels.

In order to check our DPD model, we used the DPD model to simulate Poiseuille flow with simple DPD particles within two infinitely parallel plates. There exist theoretical solutions for the Poiseuille flow. The simulation results of Poiseuille flow, can be found in our previous work (Zhou et al. 2012), which are consistent with the solutions of NS equations and the results from Fen et al. (2006). The success in modeling the Poiseuille flow can help to validate the DPD model and the choice of related parameters.

\subsection{Simple fluid flow}

Before modeling macromolecular suspension, we firstly simulated the simple fluid flow (DPD particles are not chained to form complex structures) through a grooved micro-channel. As shown in Fig. 1, the grooved micro-channel is modeling by two stationary parallel solid plates with contraction and expansion sections, characterized by periodically placed rectangular protruding bottleneck. The computational domain is $-100 \leq x \leq 100,-1.5 \leq y \leq 1.5$ and $-10 \leq z \leq 10$ while $-5 \leq z \leq 5$ at rectangular protruding bottleneck. A total number of 48996 simple DPD particles are used, including 40800 fluid particles placed in the planar slit and 8196 wall particles located in three layers parallel to the $\mathrm{x}$-y plane in each side. The periodic boundary conditions are applied to fluid boundaries in the $\mathrm{x}$ and $\mathrm{y}$ directions. On the surface of solid walls, we applied Maxwellian reflection boundary conditions to yield the no slip boundary condition.

The physical properties of a DPD fluid are characterized by the parameters in the DPD formulation. Some parameters can be determined by fitting the relevant data of the real fluid, some are chosen to maintain the numerical accuracy in simulating simple cases, such as the ideal gas and equilibrium states. For complex systems, there is no solid physical basis to determine the parameters characterized interaction strengths between different components. According to Groot and Warren's work (Goot and Warren 2007), to satisfy the compressibility of water, the coefficients of the conservation and random forces should be $a_{i j} \approx 75 k_{B} T / \rho$ and $\sigma=3.0$, with $\lambda=0.65$ in the verlet-type algorithm. This conclusion can also be extended to the dilute polymer solution (Fan et al. 2003, Fan et al. 2006). We set the unit of energy to be $k_{B} T=1$ and the density to be $\rho=4$. Thus the corresponding $a_{i j}=a_{\text {fluid }}=18.75$, if $i$ and $j$ both denote fluid particles or beads in the molecular chains. There are no physical bases on how the solid particles 


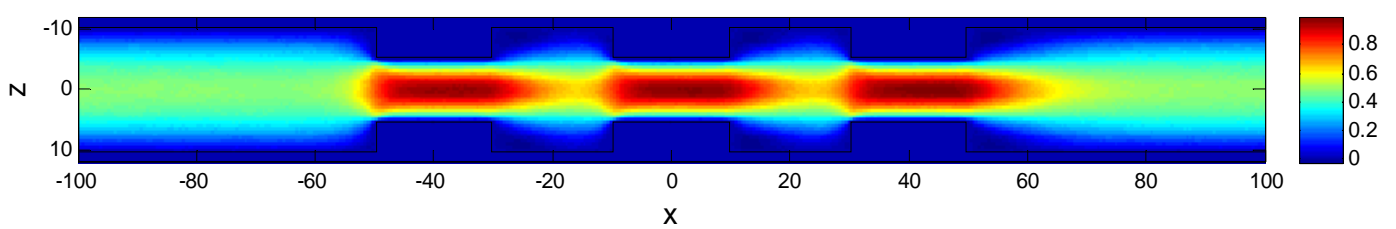

$v x$

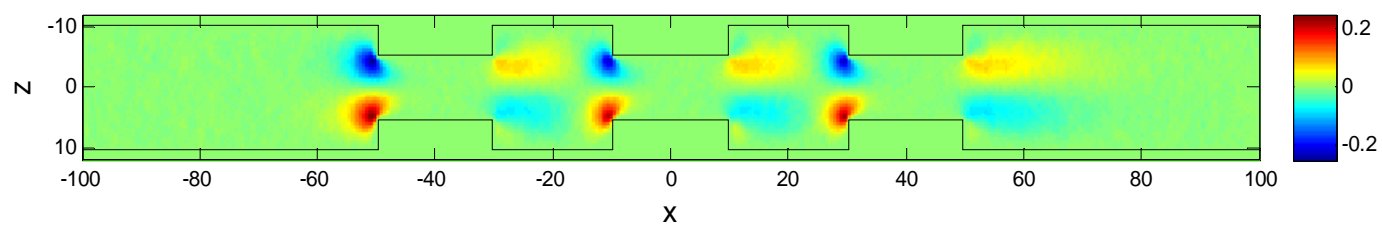

$v z$

Fig. 2 Velocity field (top: horizontal velocity $v x$; bottom: vertical velocity $v z$ )

interact with solvent particles or beads in the molecular chains. For simple, we assumed $a_{\text {wall }}=5.0$ and $a_{i j}=\sqrt{a_{\text {fluid }} a_{\text {wall }}}$ when calculating the interaction between fluid and wall particles. To generate the flow, a field force, $g=0.01$, are applied to each fluid particle and beads in the $\mathrm{x}$ direction. To solve the equation of motion, a modified velocity-verlet algorithm with time step at $\Delta t=0.01$ is used. The computational domain is divided into $400 \times 94$ bins in the $\mathrm{x}-\mathrm{Z}$ plane and local data are collected in each bin. By averaging the sampled data in each bin over 10000 time steps, we can obtain all local flow properties. For example, pressure values can be obtained from the trace of the stress tensor

$$
p=-\frac{1}{3} \operatorname{tr} \mathbf{S}
$$

where the stress tensor is calculated using the Irving-Kirkwood model (Fan et al. 2003)

$$
\mathbf{S}=-\frac{1}{V}\left[m \sum_{i}\left(\vec{v}_{i}-\vec{v}\right)\left(\vec{v}_{i}-\vec{v}\right)+\frac{1}{2} \sum_{i} \sum_{j \neq i} \vec{r}_{i j} \vec{F}_{i j}\right]
$$

In the above equation, $V$ is the volume of the computational bin, $\vec{v}$ is the corresponding stream velocity, and $\vec{F}_{i j}$ is the inter-particle force on particle $i$ due to particle $j$. If particle $i$ is a bead of molecular chains, $\vec{F}_{i j}$ should also include the total spring force on the bead. The first term of the right hand side in Eq. (13) describes the contribution to the stress from the momentum transfer of DPD particles and the second term from the inter-particle force.

Temperature is calculated in each bin using the equation

$$
T=\frac{m}{3 N k_{B}} \sum_{i=1}^{N}\left(\vec{v}_{i}-\vec{v}\right)^{2}
$$




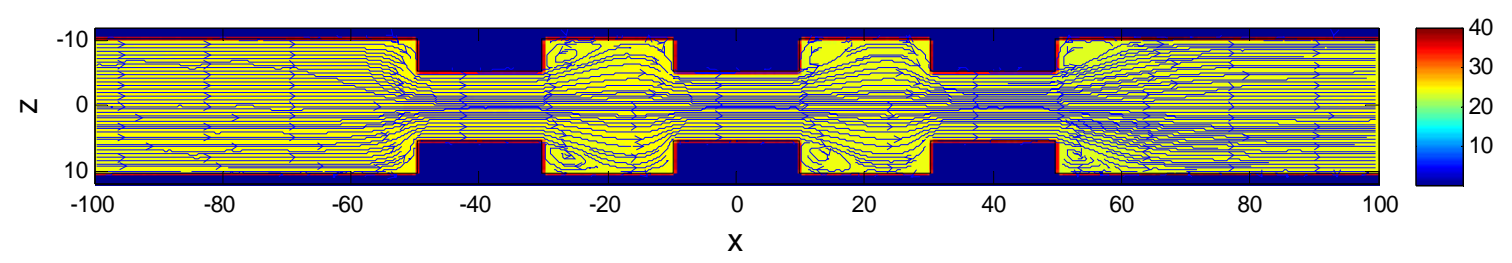

Fig. 3 The streamline of the flow field

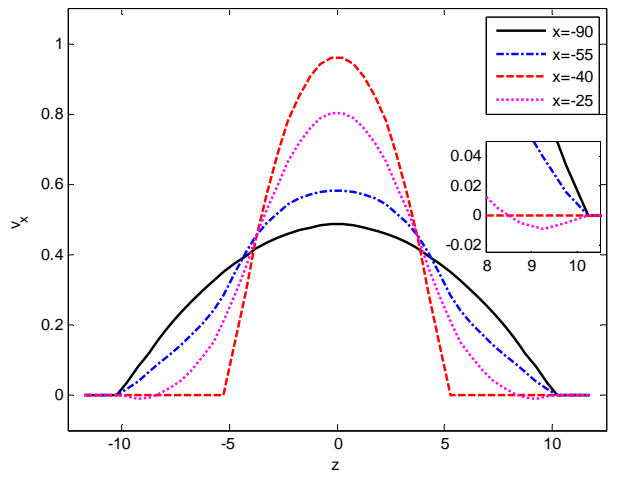

Fig. 4 The horizontal velocity profiles at $x=-90,-55,-40$ and -25

where $N$ is the number of particles inside the bin.

By averaging the velocity vectors $\left(v_{x}\right.$ and $v_{z}$ ) of all particles in each bin, we can get the velocity field. Fig. 2 shows the velocity field in $x$ and $y$ direction respectively. It is easy to get the streamline of the flow field as we have already got the velocity of all bins. Fig. 3 shows the streamline of the flow field. It is observed that the horizontal velocity is smaller in the region near the wall. As the width of the channel reduces, the horizontal velocity increases.

In areas with a sudden contraction, for example, flow contracts from cross-section $x=-60$ to cross-section $x=-40$, forming a vena contraction. This contraction rate is about $50 \%$ (half of the original channel width). In a sudden expansion section, for example, fluid flow from cross-section $x=-40$ to cross-section $x=-20$, the horizontal velocity gradually reduces. It is observed that vortices have been generated at the top and bottom corners behind the contraction structure (e.g. $-30 \leq x \leq-20$ ).

In order to better show the difference of horizontal velocity at different position, the profiles of horizontal velocity at $x=-90,-55,-40$ and -25 are plotted in Fig. 4 . The horizontal velocity profile at $x$ is calculated by average the local horizontal velocity of bins between $x-5$ and $x+5$ along the $x$ direction. For example, the profile of horizontal velocity at $x=-90$ is actually mean value of horizontal velocity between $x=-95$ and $x=-85$ along the $\mathrm{x}$ direction.

It is found in Fig. 4 that the structure of the grooved channel with sudden contraction and expansion strongly affects the velocity distribution. There are obvious differences in the velocity distribution of different cross-sections. The horizontal velocity profile at $x=-90$, far away from sudden contraction and sudden enlargement, is close to quadratic. This indicates that the flow at $x=-90$ is close to Poiseuille flow. The horizontal velocity profile at $x=-55$, facing a sudden 


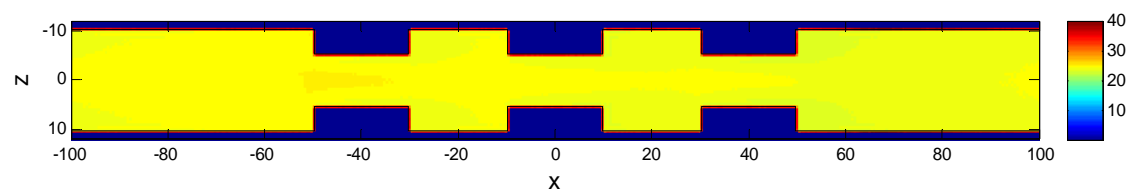

Pressure field

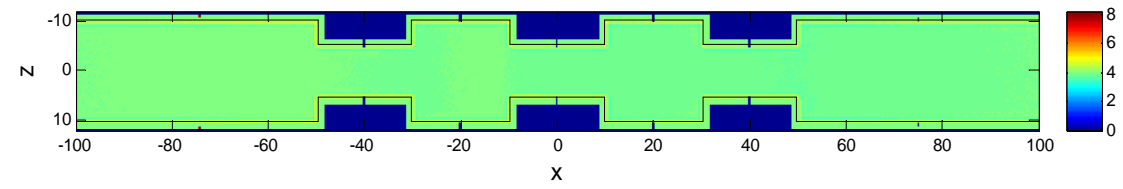

Number density field

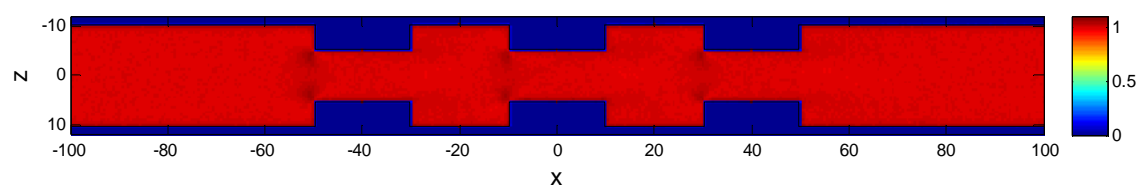

Temperature field

Fig. 5 Pressure (top), density (middle) and temperature (bottom) field

contraction, is higher in center but lower in marginal regions. The horizontal velocity profile at $x=-40$, in the center region of the contraction section, is close to quadratic too but is much sharper than the profile at $x=-90$. The horizontal velocity profile at $x=-25$, just behind a sudden enlargement, is negative in marginal region, which correspond to the vortices in Fig. 3. It is a coincidence that velocity profiles at $x=-55,-40$ and -25 intersect at two points.

Local pressure, number density, temperature and streaming velocity are also calculated by solving the Eqs. (13) and (14). Fig. 5 shows the pressure, number density and temperature field. Pressure, number density, temperature are almost uniform across the channel.

Similarly, the profiles of density and temperature at $x=-90,-55,-40$ and -25 are also plotted to show the difference of local properties at different cross-sections (see Fig. 6).

Form Fig. 6, we can see that temperature is almost uniform across the channel at each cross-section except for regions near the wall. The temperature drops near the wall due to low wall temperature. Density is uniform in most regions except for marginal regions near the wall, where density fluctuations exist. The peak density value near the wall is higher than that in the center of the channel due to mass conservation. These fluctuations in density are not only due to the interaction of fluid particles and wall particles, but also due to bounce-back conditions. The structure of quadrate contraction has slight influences on the density distribution at different cross-sections. We can easily find that the density of particles in the region just facing the contraction construct is slight higher than other regions. It may be due to the quadrate contraction blocking the flow from the left to the right.

The density profile at cavity $(x=-25)$ is nearly constant and almost coincide with the profile at $x=-90$ far away from protrusion and cavity. But it should be noted that a small difference exists between the two profiles for regions very close the wall. This phenomenon may be caused by particle trapping inside the cavities of the vortices. To confirm this assumption, we investigate the particle trajectories. 


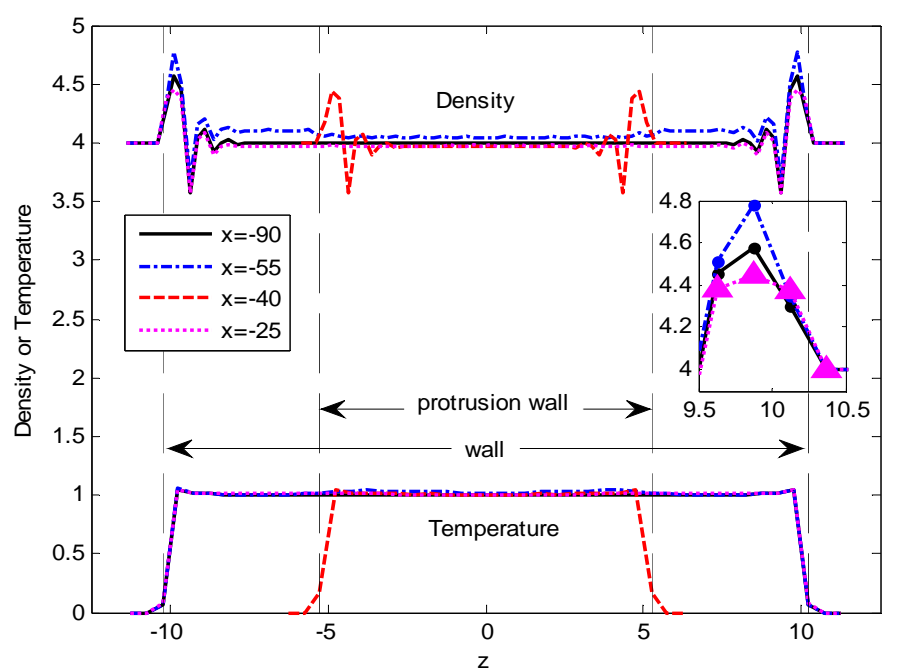

Fig. 6 Density and temperature profiles at different $x$ across the slit

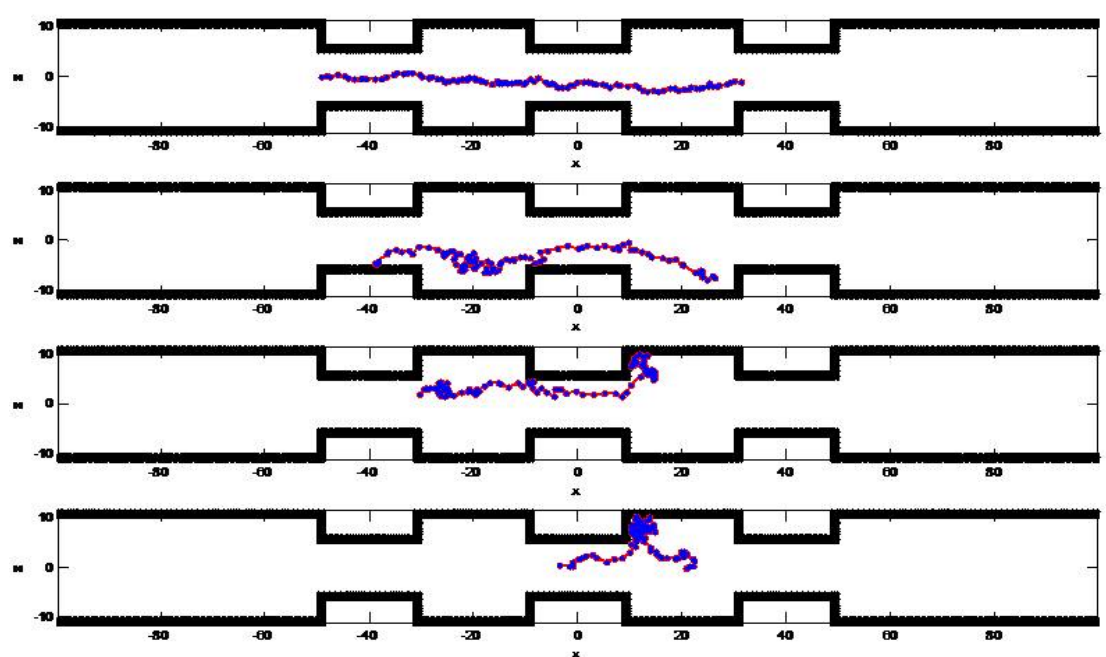

Fig. 7 Four typical trajectories of particles

The trajectory of particles in DPD simulation does not strictly flow along the streamline due to the random force. Fig. 7 shows four typical trajectories of four concerned particles at different instants:

- The first trajectory shows a particle through the region with protrusions and cavities very smoothly without any block. These trajectories usually exist in the center region of $z$ direction.

- The second trajectory shows a particle through the region with protrusions and cavities smoothly except in the cavity $(-30 \leq x \leq-10)$. The particle hesitates in the cavity for a significant amount of time. These trajectories usually exist in the region which is close to the protrusion wall. 


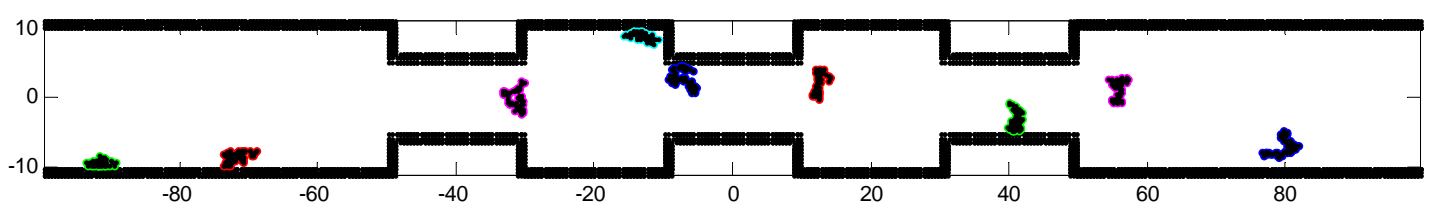

(a) $t=0$

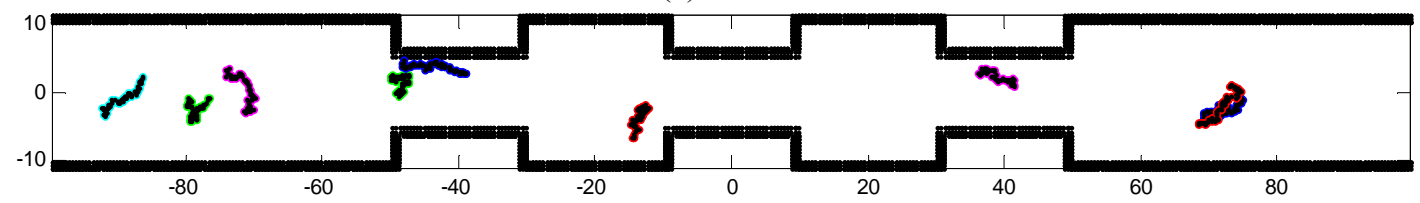

(b) $t=8000$

Fig. 8 The initial configures and final conformations of macromolecules in the grooved micro-channel

- The third trajectory shows a particle is trapped by the vortices inside the cavity after through two protrusions and one cavity. These trajectories usually exist in the region very close to the protrusion wall.

- The fourth trajectory shows a particle escaping from the vortices inside the cavity after being trapped. This trajectory is the extension from the third one. A detailed trajectory analysis shows that there are no particles that stay trapped inside the cavities for the whole simulation time. This phenomenon indicates that this trapping effect does not depend on the initial particles configuration. Particles that are initially located inside the cavities do not stay there during the entire simulation. Meanwhile, particles that are initially distributed outside the cavities may enter the cavity and are trapped during the simulation.

\subsection{Movement and evolution of macromolecules}

In this section, we use DPD particles and FENE chains to model the suspension of macromolecules by just adding FENE chains to the flow field of grooved micro-channel flow. 40500 fluid particles and 1800 FENE chain beads (30 FENE chains each with 60 beads) are used in the simulations to model the dynamic behavior of macromolecular suspension in grooved micro-channel. The parameters in the spring force law of FENE are taken to be $H=6.0, r_{m}=1.5$. Other parameters remain the same as those mentioned in the previous simulation.

Fig. 8(a) shows the initial configurations of some selected macromolecules in the grooved micro-channel. In the initial moment $(t=0)$, all chains randomly and relatively uniform distributed in the entire flow field, and most of them are coiled.

As flow develops, the FENE chains move, rotate and stretch. When fully developed $(t=8000)$, as shown in Fig. 8(b), most chains are distributed in regions between $z=-5$ and $z=5$, Macromolecules located nearer to walls are stretched better than those in the center region, where macromolecules are usually coiled. The coiled chains are found in the central part of the slit, where shear rate is low, while the longest chains are found in the region close to wall, where shear rate is high.

Fig. 9 shows the velocity field and Fig. 10 shows the streamline of the flow field. From Figs. 9 and 10, the movement and evolution of macromolecules with FENE chain do not show significant difference in velocity fields and streamline, compared with those from the simple flow. 


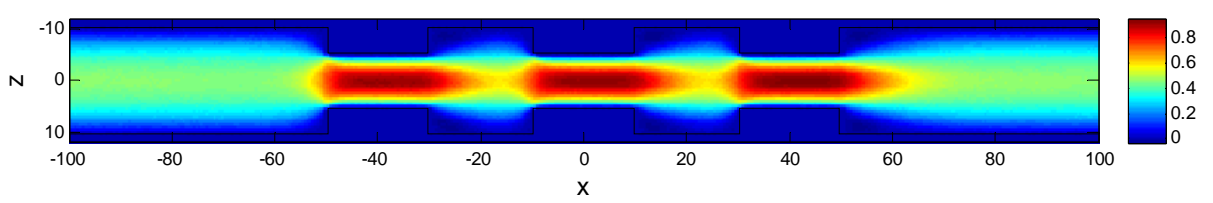

$v x$

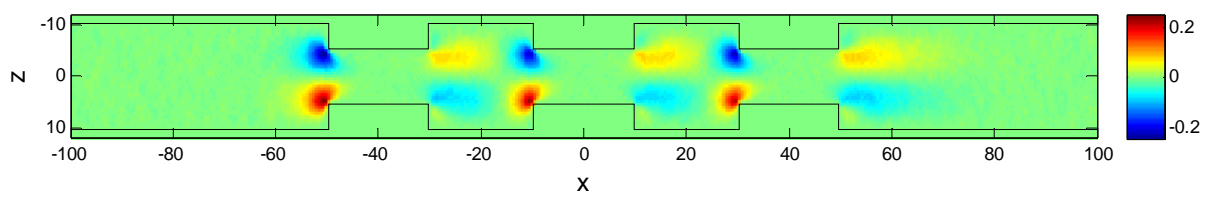

$v z$

Fig. 9 Velocity field (top: horizontal velocity $v x$; bottom: vertical velocity $v z$ )

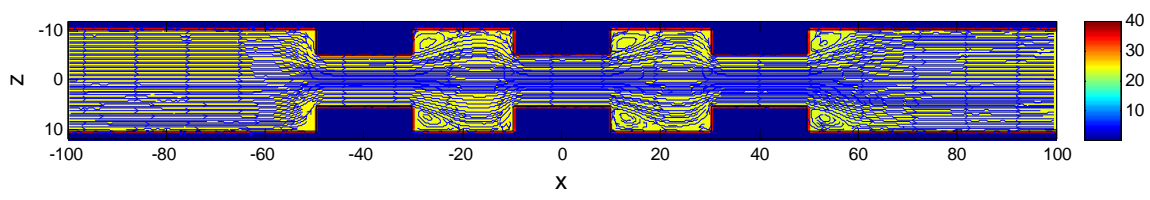

Fig. 10 Streamline of the flow field

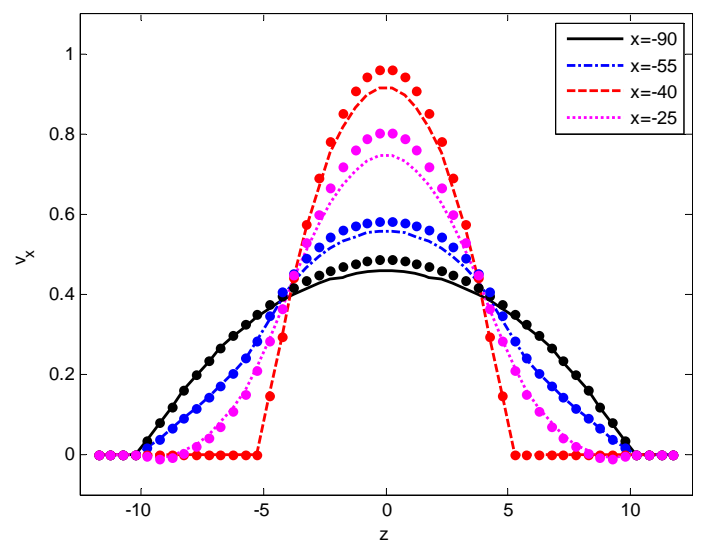

Fig. 11 Velocity, profiles at different $x$. (Dots indicate the velocity profiles of simple DPD fluid flow)

It is not easy for us to identify the difference properties between flow of macromolecules suspension and flow of simple fluid by directly observing the flow fields. Hence, we draw the profiles of horizontal velocity at $x=-90,-55,-40$ and -25 (see Fig. 11). In Fig. 11, lines indicate the horizontal velocity profiles from macromolecules suspension, while dots indicate those from simple fluid flow.

From Fig. 11, we can see that macromolecular chains have remarkable influences on the velocity distribution across the micro-channel. The existence of macromolecular chains will drop velocity in center region of the channel. It is observed that the impact of the macromolecular 


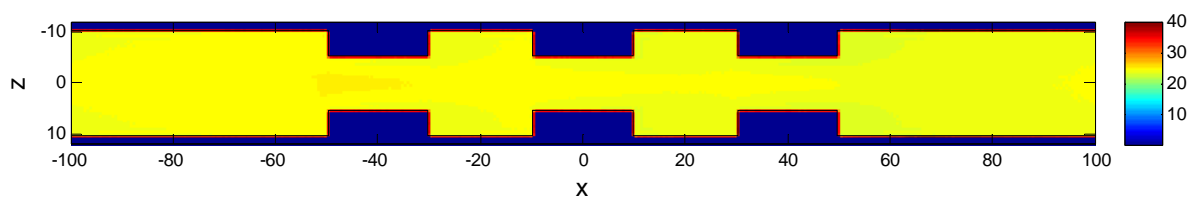

Pressure field

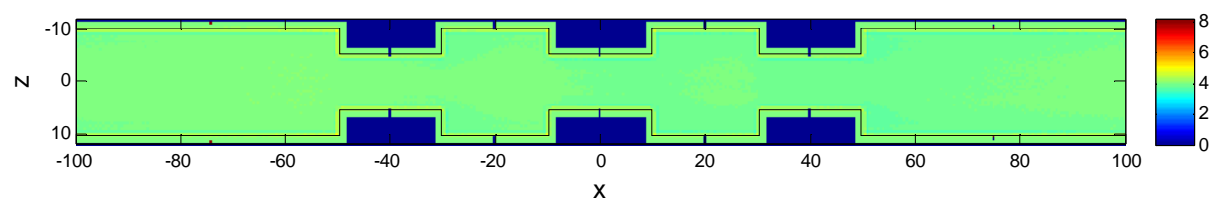

Number density field

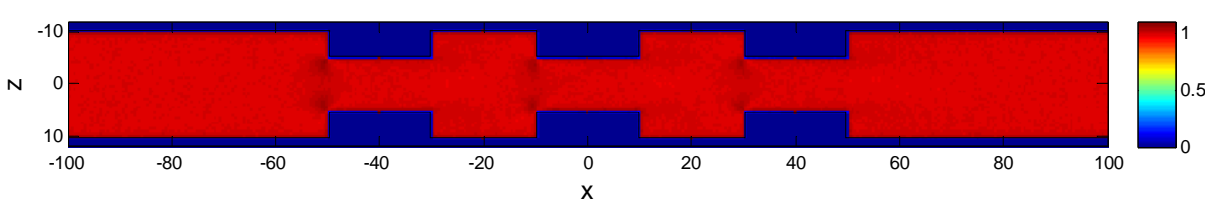

Temperature field

Fig. 12 Pressure (top), density (middle) and temperature (bottom) field

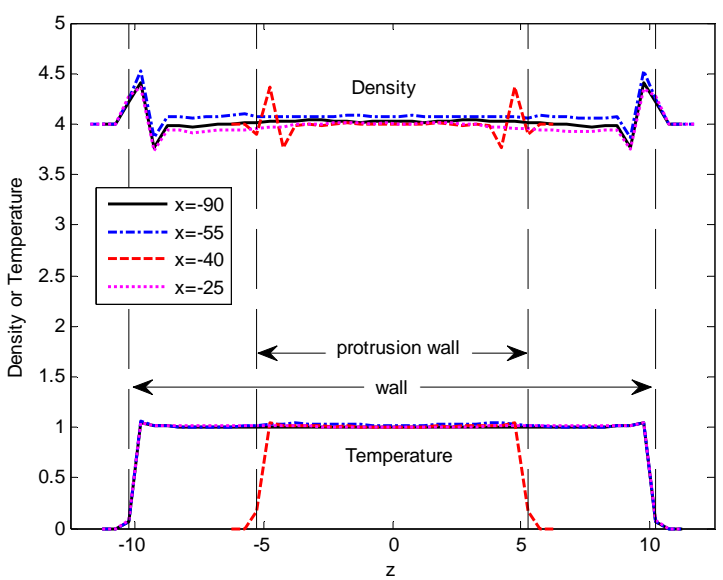

Fig. 13 Density and temperature profiles at different $\mathrm{x}$ across the slit

chains on the velocity distribution is more significant in the region $-5 \leq z \leq 5$, and this result is consistent with the phenomenon that most chains are distributed in the region $-5 \leq z \leq 5$ as shown in Fig. 8.

Fig. 12 shows the pressure, number density and temperature field of macromolecules suspension flow. Similar to the simple fluid flow, pressure, number density and temperature are almost uniform in the channel except for some areas close to walls.

We also draw the profiles of density and temperature at $x=-90,-55,-40$ and -25 as shown in Fig. 13. We can see the existence of macromolecule chains do not significantly influence the temperature distribution: the temperature is almost uniform across the channel and is very close to 

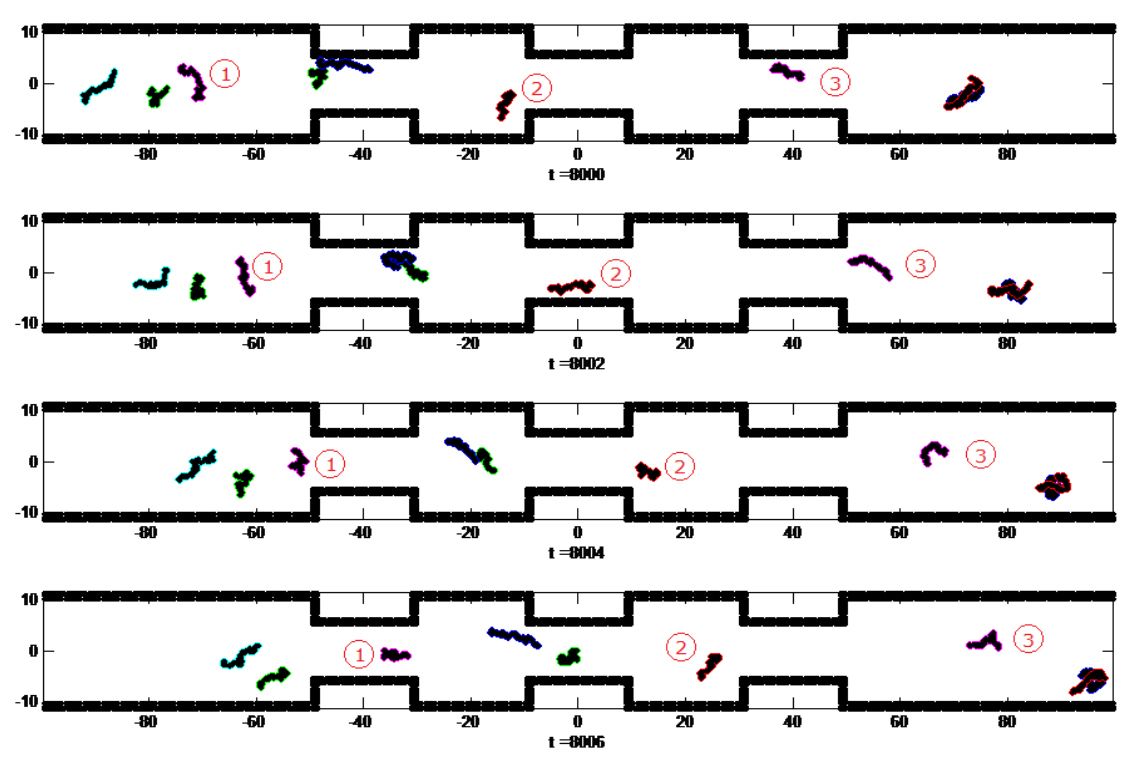

Fig. 14 Conformation evolution of marked macromolecules passing through the channel

the given temperature, but only drops near the wall due to low wall temperature. Meanwhile, macromolecular chains have slight influence on the density distribution across the micro-channel: the density of central region is slightly higher instead of uniform across the channel. Region where macromolecular chains are relatively dense has a slight higher particles density. Whether there exists macromolecules or not, a fluctuation in density exists in the regions near the wall.

In order to observe the dynamic behavior of macromolecules passing through the channel, serial frames of cconformations of marked macromolecules in the channel are plotted in Fig. 14. It is observed that macromolecules kept changing their own conformation due to the random force in DPD formula, which corresponds to the thermal fluctuations in mesoscopic scale. From the conformations of a single macromolecule in the serial frame, we can find that macromolecule trend to stretch longer when passing the region close to wall than middle region of the channel. The structure of the grooved channel with sudden contraction and enlargement affect the conformation evolution of the macromolecule significantly. To better illustrate this effect, we labeled each macromolecule in Fig. 14. When facing a sudden contraction, macromolecule tends to coil itself, or adjust the direction of extension to parallel the protruding wall, to pass the channel smoothly. For example, Macromolecule \# 1 changes from a stretching state to becomecurled before and close to the contraction section. Macromolecule \# 2 changes from a vertically stretching state to become a horizontal stretching state, and become curled before, in and after the contraction section. When facing a sudden enlargement, macromolecule can stretch and rotate, just as shown by Macromolecule \# 3. A detailed conformation and trajectory observation of macromolecule show that there is no macromolecule trapped by the vortices inside the cavity for the whole simulation time, while simple DPD particle can be trapped by the vortices. Therefore, it is reasonable to draw such a conclusion: macromolecule would like to adjust its own conformation and angle to suit the flow and pass the channel quickly. 


\section{Conclusions}

In this paper, we investigated the transport and conformation of macromolecules in a grooved micro-channel by using the dissipative particle dynamics model with finitely extendible non-linear elastic bead spring chains model. The grooved micro-channel is characterized by periodically placed rectangular protruding bottleneck. We firstly use the DPD model to simulate the simple fluid flow in the grooved micro-channel. The flow fields and profiles were analyzed. We found that the horizontal velocity is smaller in the region near the wall. As the width of the channel reduces, the horizontal velocity increases simultaneously. The structure of the grooved channel with sudden contraction and expansion strongly affects the velocity distribution. Vertices can also be found at the top and bottom corners behind the contraction section. The particle trajectories were also investigated by tracking four typical trajectories of particles in detail. It is found that particles can be trapped or escape from the vertices, while no particles trapped inside the cavities for the whole simulation time.

After the successful simulation of simple DPD fluid in the grooved micro-channel, we use DPD particles with FENE chains to model the movement and evolution of macromolecules in grooved micro-channel. Our numerical results show that macromolecular chains influence velocity and density distribution rather than the temperature and pressure. Macromolecular chains have remarkable influence on the velocity distribution across the channel. The existence of macromolecular chains will reduce velocity in the center region of the channel where most chains were found. Macromolecular chains have slight influences on the density distribution across the channel. The density in the central region is slightly higher instead of uniform across the channel. For regions where macromolecular chains are relatively dense, the associated particle density is slightly higher. It can be concluded that macromolecules tend to drag simple fluid particles, reducing their velocity, and leading to density and velocity fluctuations. We also analyzed the cconformation evolution of macromolecules passing through the channel, and conclude that macromolecules are able to adjust their own conformations to suit the shape of geometric structures and pass the channel smoothly.

\section{Acknowledgements}

This work has been supported by the National Natural Science Foundation of China (50976108), the 100 Talents Programme of the Chinese Academy of Sciences (A1041) and the Shanxi International Collaboration Project (2011081040).

\section{References}

Brazzle, J.D., Mohanty, S. and Frazier, A.B. (1999), "Hollow metallicmicromachined needles with multiple output ports”, Conf. Microfluidic Device Systems, 3877, 257-266.

Chen, S. and Doolen, G.E. (1998), "Lattice-boltzmann method for fluid flows", Annu. Rev. Fluid Mech., 30, 329-364.

Chu, S. (1991), "Laser manipulation of atoms and particles", Science, 253, 861-866.

Chun, K., Hashiguchi, G., Toshiyoshi, H. and Fujita, H. (1999), "Fabrication of array of hollow microcapillaries used for injection of genetic materials into animal/plant cells", Jpn. J. Appl. Phys., 38(2), 279-281. 
Doyle, P.S. and Shaqfeh, E.S.G. (1998), "Dynamics simulation of freely draining, flexible bead-rod chains: start-up of extensional flow", J. Non-Newton. Fluid Mech., 76(1), 43-78.

Doyle, P.S. and Shaqfeh, E.S.G. (1998), "Dynamics simulation of freely-draining, flexible bead-rod chains: startup of extensional flow", J. Non-Newton. Fluid Mech., 76(1), 43-78.

Doyle, P.S., Shaqfeh, E.S.G. and Gast, A.P. (1997), "Dynamics simulation of freely-draining, flexible polymers in steady linear flows", J. Fluid Mech., 334(1), 251-291.

Doyle, P.S., Shaqfeh, E.S.G., McKinley, G.H. and Spiegelberg, S.H. (1998), "Relaxation of fillet polymer-solutions following extensional flow", J. Non-Newton. Fluid Mech., 76(1), 79-110.

Duong-Hong, D., Wang, J.S., Liu, G.R., Chen, Y.Z., Han, J. and Hadjiconstantinou, Nicolas G. (2008), "Dissipative particle dynamics simulations of electroosmotic flowin nano-fluidic devices", Microfluid Nanofluid, 4(3), 219-225.

Español, P. and Warren, P. (1995), "Statistical mechanics of dissipative particle dynamics", Europhys. Lett., 30(4), 191-196.

Fan, X., Phan-Thien, N., Yong, N.T., Wu, X. and Xu, D. (2003), "Microchannel flow of a macromolecular suspension", Phys. Fluids, 15(1), 11-21.

Fan, X., Nhan, P.T., Chen, S., Wu, X. and Ng, T.Y. (2006), "Simulating flow of DNA suspension using dissipative particle dynamics", Phys. Fluids, 18(6), 063102.

Frisch, U., Hasslachcher, B. and Pomeau, Y. (1986), "Lattice-gas automata for the navier-stokes equation", Phys. Rev. Lett., 56(14), 1505-1508.

Groot, R.D. and Warren, P.B. (1997), "Dissipative particle dynamics: Bridging the gap between atomistic and mesoscopic simulation", J. Chem. Phys., 107(11), 4423-4435.

Hoogerbrugge, P.J. and Koelman, J.M.V.A. (1992), "Simulating microscopic hydrodynamic phenomena with dissipative particle dynamics", Europhys. Lett., 19(3), 155-160.

Hur, J.S., Shaqfeh, E.S.G. and Larson, R.G. (2000), "Brownian dynamics simulation of single DNA molecules in shear flow", J. Rheol., 44(4), 713-743.

Kasiteropoulou, D., Karakasidis, T.E. and Liakopoulos, A. (2011), “A dissipative particle dynamics study of flow in periodically grooved nanochannels", Int. J. Numer. Meth. Fluids, 68(9), 1156-1172.

Lin, L. and Pisano, A.P. (1999), "Silicon processed microneedles", IEEE J. Micromech.Syst., 8(1), 78-84.

Pan, H., Ng, T.Y., Moeendarbary, H. and Li, E. (2010), "Dissipative particle dynamics simulation of entropic trapping for DNA separation", Sensor. Actuat., 157(2), 328-335.

Perkins, T.T., Quake, S.R., Smith, D.E. and Chu, S. (1994), "Relaxation of single DNA molecule observed by optical microscopy", Science, 264, 822-826.

Perkins, T.T., Smith, D.E. and Chu, S. (1994), "Direct observation of tube-like motion of a single polymer chain", Science, 264, 819-822.

Perkins, T.T., Smith, D.E. and Chu, S. (1997), "Single polymer dynamics in an elongational flow", Science, 276, 2016-2080.

Perkins, T.T., Smith, D.E., Larson, R.G. and Chu, S. (1995), "Stretching of a single tethered polymer in a uniform flow", Science, 268, 83-87.

Shrewsbury, P.J., Muller, S.J. and Liepmann, D. (2001), "Effect of flow on complex biological macromolecules in microfluidic devices", Biomed. Microdevices, 3(3), 225-238.

Smith, D.E., Babcock, H.P. and Chu, S. (1999), "Single-polymer dynamics in steady shear flow", Science, 283(5408), 1724-1727.

Smith, D.E. and Chu, S. (1998), "Response of flexible polymers to sudden elongation flow", Science, 281(5381), 1335-1340.

Smith, S.B., Finzi, L. and Bustamante, C. (1992), "Direct mechanical measurements of the elasticity of single DNA molecules”, Science, 258, 1122-1126. 